\title{
Magnetic Properties of NpPdSn
}

\author{
K. Gofryk*, J.-C. Griveau, R. Jardin, E. Colineau, J. Rebizant, F. Wastin \\ and R. Caciuffo \\ European Commission, Joint Research Centre \\ Institute for Transuranium Elements \\ Postfach 2340, 76125 Karlsruhe, Germany
}

\begin{abstract}
A new compound NpPdSn was prepared and studied by X-ray diffraction, magnetization, heat capacity and electrical resistivity measurements, performed in the temperature range $2-300 \mathrm{~K}$ and under magnetic field up to $14 \mathrm{~T}$. The crystal structure determined by single-crystal X-ray analysis is hexagonal with ZrNiAl-type (space group $P \overline{6} 2 \mathrm{~m})$. NpPdSn orders antiferromagnetically at $19 \mathrm{~K}$ and exhibits a Curie-Weiss behavior with $\mu_{\mathrm{eff}}=2.66 \mu_{\mathrm{B}}$ and $\Theta_{\mathrm{p}}=-47 \mathrm{~K}$. Bulk properties show temperature variations similar to systems with strong electronic correlations with a large negative paramagnetic Curie temperature and an enhanced low-temperature specific heat $\left(\gamma \approx 90 \mathrm{~mJ} /\left(\mathrm{mol} \mathrm{K}{ }^{2}\right)\right)$. It suggests that NpPdSn may be classified as a new Np-based antiferromagnetic Kondo lattice, one of the very few known amidst transuranium-based intermetallics.
\end{abstract}

PACS numbers: 75.50.Ee, 71.27.+a, 75.30.Mb

\section{Introduction}

Uranium-based compounds with the UTX composition, where $\mathrm{T}$ is a $d$-electron transition metal and $\mathrm{X}$ stands for a $p$-electron element, have attracted much attention for their large variety of physical behaviors, due to the hybridization of uranium $5 f$ electrons with $s, p$ and/or $d$ electrons of neighboring atoms. In these ternaries itinerant or localized magnetic moment, complex magnetic structures and/or semiconducting or semimetallic-like electrical conductivity have been reported. Moreover, several UTX compounds also show features characteristic of heavy fermion systems. Such a behavior was reported, for example, for magnetically ordered Kondo lattices such as UPdGa (two subsequent antiferromagnetic-like transitions at 30 and $62 \mathrm{~K}[1]$ ), UPdSi (antiferromagnetic-like transitions at 27 and $33 \mathrm{~K} \mathrm{[2]),} \mathrm{UPdGe} \mathrm{(antiferromagnetic} \mathrm{transition}$ at $50 \mathrm{~K}$ and a ferromagnetic one at $28 \mathrm{~K}[3,4]$ ) and UPdIn (antiferromagnetic ordering below $T_{\mathrm{N}}=20 \mathrm{~K}$ with a ferromagnetic component below $7 \mathrm{~K}[5]$ ). The uranium stannite, UPdSn, exhibits well localized $5 f$ electrons with a small linear specific heat coefficient $(\gamma \approx$ $\left.5 \mathrm{~mJ} /\left(\mathrm{mol} \mathrm{K}^{2}\right)\right)[6]$. This compound exhibits an antiferromagnetic phase transition at $37 \mathrm{~K}$ leading to a magnetic structure with orthorhombic symmetry. A second transition occurs at $25 \mathrm{~K}$, and the magnetic structure becomes of monoclinic symmetry [7]. The ordered magnetic moment on the $\mathrm{U}$ site is large $\left(\mu_{\text {ord }} \approx 2 \mu_{\mathrm{B}}\right)$ [8].

In the course of our systematic studies on the magnetic, transport and thermodynamic properties of transuranium-based $\mathrm{AnPd}_{2} \mathrm{Sn}$ intermetallics [9-11], we

\footnotetext{
* corresponding author; e-mail: krzysztof.gofryk@ec.europa.eu
}

have recently focused our attention on the AnPdSn materials. In this paper we report for the first time about crystal structure, magnetic and specific heat properties of NpPdSn.

\section{Experimental details}

A polycrystalline sample of $\mathrm{NpPdSn}$ was prepared by arc melting the stoichiometric amounts of the elements in a Zr-gettered pure argon atmosphere. The sample was examined by X-ray single crystal and powder diffraction methods. Small single crystals were isolated from the

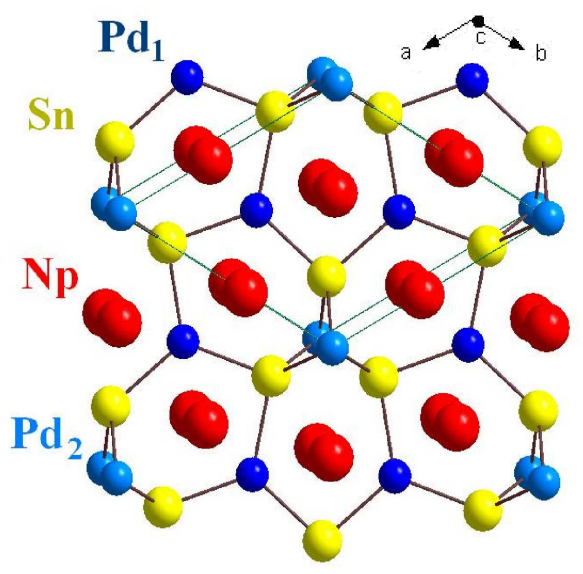

Fig. 1. Crystal structure of NpPdSn.

crushed ingot and used for the data collection on a four-circle X-ray diffractometer. The refined crystal structure was shown to be hexagonal with ZrNiAl-type (space 
group $P \overline{6} 2 m)$ and with lattice parameters $a=7.5076 \AA$ and $c=4.0954 \AA$. A sketch of the crystal structure of NpPdSn is shown in Fig. 1.

Magnetization measurements were carried out in the temperature range $2-300 \mathrm{~K}$ and in magnetic fields up to $7 \mathrm{~T}$ using a SQUID magnetometer (Quantum Design MPMS-7). The heat capacity (relaxation method) was measured from 2 to $300 \mathrm{~K}$ employing a Quantum Design PPMS-14 system. Due to the radiotoxicity of neptunium, all operations of preparation and encapsulation have been achieved in glove boxes under inert atmosphere $\left(\mathrm{N}_{2}\right)$. Moreover, all experimental studies were made using special encapsulation systems due to the contamination risks.

\section{Results and discussion}

The temperature dependence of the inverse magnetic susceptibility of NpPdSn measured in an applied magnetic field of $7 \mathrm{~T}$ is shown in Fig. 2. At low temperature a distinct minimum in the $\chi^{-1}(T)$ curve manifests the onset of antiferromagnetic ordering below the Néel temperature $T_{\mathrm{N}}=19 \mathrm{~K}$.

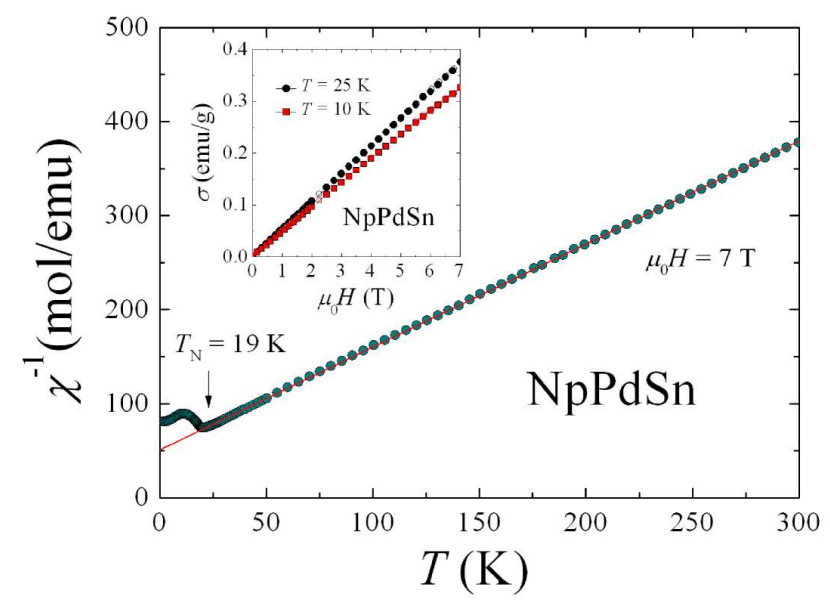

Fig. 2. Temperature dependence of the inverse magnetic susceptibility of NpPdSn measured in a magnetic field $\mu_{0} H=7 \mathrm{~T}$. The solid line is a Curie-Weiss fit. Inset: magnetization versus magnetic field taken at 10 and $25 \mathrm{~K}$.

Above the magnetic transition, the inverse magnetic susceptibility of NpPdSn follows a Curie-Weiss law:

$$
\chi(T)=\frac{N \mu_{\mathrm{eff}}^{2}}{3 k_{\mathrm{B}}\left(T-\Theta_{\mathrm{p}}\right)}
$$

with the effective magnetic moment $\mu_{\text {eff }}=2.66 \mu_{\mathrm{B}}$ and the paramagnetic Curie temperature $\Theta_{\mathrm{p}}=-47 \mathrm{~K}$. The experimental value of $\mu_{\text {eff }}$ is similar to the free $\mathrm{Np}^{3+}$ ion value expected for the Russell-Saunders coupling $\left(2.68 \mu_{\mathrm{B}}\right)$. It indicates the presence of a well localized magnetic moment on the $\mathrm{Np}$ ions. The absolute value of $\Theta_{\mathrm{p}}$ is much larger compared to the Néel temperature as usually found in systems with strong Kondo interactions. As shown in the inset of Fig. 2, the magnetization measured in the ordered state is proportional to the applied magnetic field with no sign of any hysteresis effect or metamagnetic-like transition up to $7 \mathrm{~T}$.

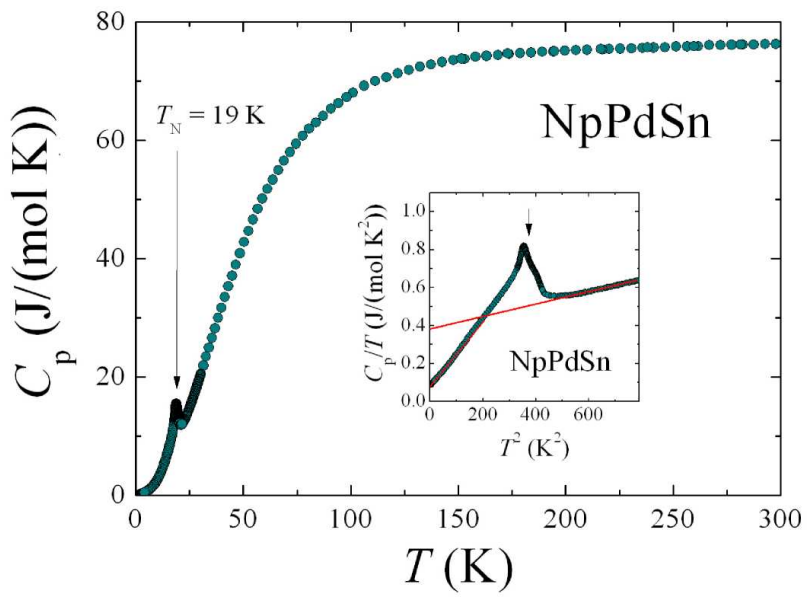

Fig. 3. Temperature variation of the specific heat of NpPdSn. The inset shows the low-temperature data in the form $C_{p} / T$ vs. $T^{2}$. The solid lines mark a straight line behavior.

Figure 3 shows the temperature dependence of the specific heat of NpPdSn. At room temperature, $C_{p}$ approaches a value expected from the Dulong-Petit law, i.e. $C_{p}=3 n R=74.8 \mathrm{~J} /(\mathrm{mol} \mathrm{K})$, where $n$ is the number of atoms per molecule (in the case of $\mathrm{NpPdSn} n=3$ ) and $R$ is the gas constant. The magnetic phase transition at $T_{\mathrm{N}}=19 \mathrm{~K}$ manifests itself as a small $\lambda$-type anomaly in $C_{p}(T)$. This peak is hardly affected by an applied magnetic field. At $14 \mathrm{~T}$, the maximum in $C_{p} / T$ shifts only faintly towards lower temperatures and insignificantly decreases in magnitude. The observed behavior is very similar to that found before in $\mathrm{NpPd}_{2} \mathrm{Sn}$ [9]. The low temperature electronic contribution to the specific heat of NpPdSn is strongly enhanced. The $C_{p} / T$ ratio extrapolated to $T=0 \mathrm{~K}$ from the regions above and below $T_{\mathrm{N}}$ is as large as $380 \mathrm{~mJ} /\left(\mathrm{mol} \mathrm{K}^{-2}\right)$ and $90 \mathrm{~mJ} /\left(\mathrm{mol} \mathrm{K}^{-2}\right)$, respectively (see inset in Fig. 3). These values are comparable with the Sommerfeld coefficients reported for UPdSb and UPdIn, which were $62 \mathrm{~mJ} /\left(\mathrm{mol} \mathrm{K}^{-2}\right)$ [12] and $280 \mathrm{~mJ} /\left(\mathrm{mol} \mathrm{K}^{-2}\right)$ [13], respectively.

\section{Summary}

The new Np-based ternary compound NpPdSn was synthesized and studied by X-ray diffraction, magnetization and heat capacity measurements, performed in the temperature range $2-300 \mathrm{~K}$ and in magnetic field up to $14 \mathrm{~T}$. The compound crystallizes with hexagonal structure of $\mathrm{ZrNiAl}$-type (e.g. $P \overline{6} 2 m$ ) with the lattice parameters: $a=7.5076 \AA$ and $c=4.0954 \AA$. The magnetic measurements revealed that NpPdSn orders antiferromagnetically at $19 \mathrm{~K}$. The enhanced low-temperature linear specific heat coefficient points to the presence of heavy-quasiparticles in the antiferromagnetic state. It suggests 
that the compound may be classified as a new Np-based antiferromagnetic Kondo lattice, one of the very few known amidst transuranium-based intermetallics. However, before drawing any firm conclusion about the electronic ground state in NpPdSn future experimental studies are required. Some of them are presently underway.

\section{Acknowledgments}

We thank D. Bouëxière for technical assistance. High purity Np metal was made available through a loan agreement between Lawrence Livermore National Laboratory and ITU, in the frame of a collaboration involving LLNL, Los Alamos National Laboratory and the US Department of Energy. K.G. acknowledges the European Commission for support in the frame of the "Training and Mobility of Researchers" program.

\section{References}

[1] V.H. Tran, R. Troć, Less-Common Met. 175, 267 (1991).

[2] K. Prokeš, V. Sechovsky, R.A. Robinson, R. Sonntag, P. Svoboda, F. de Boer, Physica B 241-243, 687 (1997).

[3] V.H. Tran, R. Troć, D. Badurski, J. Magn. Magn. Mater. 87, 291 (1990).

[4] S. Kawamata, K. Ishimoto, H. Iwasaki, H. Kobayashi, Y. Yamaguchi, T. Komatsubara, G. Kido, T. Mitsugashira, Y. Muto, J. Magn. Magn. Mater. 90-91, 513 (1990).
[5] V.H. Tran, R. Troć, J. Magn. Magn. Mater. 88, 287 (1991).

[6] F.R. de Boer, E. Brück, H. Nakotte, A.V. Andreev, V. Sechovsky, L. Havela, P. Nozar, C.J.M. Denissen, K.H.J. Buschow, B. Vaziri, M. Meissner, H. Maletta, P. Rogl, Physica B 176, 275 (1992).

[7] H. Nakotte, R.A. Robinson, A. Purwanto, Z. Tun, K. Prokeš, E. Brück, F.R. de Boer, Phys. Rev. B 58, 9269 (1998).

[8] R.A. Robinson, A.C. Lawson, J.W. Lynn, K.H.J. Buschow, Phys. Rev. B 45, 2939 (1992).

[9] D. Kaczorowski, K. Gofryk, P. Boulet, J. Rebizant, P. Javorský, E. Colineau, F. Wastin, G.H. Lander, Physica B 359-361, 1102 (2005).

[10] K. Gofryk, D. Kaczorowski, E. Colineau, F. Wastin, R. Jardin, J.-C. Griveau, N. Magnani, J. Rebizant, P. Boulet, P. Javorský, R. Caciuffo, Physica B 403, 847 (2008).

[11] K. Gofryk, D. Kaczorowski, J.-C. Griveau, N. Magnani, R. Jardin, E. Colineau, J. Rebizant, F. Wastin, R. Caciuffo, Phys. Rev. B 77, 014431 (2008).

[12] T.T.M. Palstra, G.J. Nieuwenhuys, R.F.M. Vlastuin, J. van den Berg, J.A. Mydosh, K.H.J. Buschow, J. Magn. Magn. Mater. 67, 331 (1987).

[13] E. Brück, F.R. de Boer, V. Sechovsky, L. Havela, Europhys. Lett. 7, 177 (1988). 